\begin{tabular}{|c|l|}
\hline Title & On the motion by singular interfacial energy \\
\hline Author(s) & Giga, Y.; Paolini, M.; Rybka, P. \\
\hline Citation & Hokkaido University Preprint Series in Mathematics, 498, 1-21 \\
\hline Issue Date & 2000-11-1 \\
\hline DOI & 10.14943/83644 \\
\hline Doc URL & http://hdl.handle.net/2115/69248 \\
\hline Type & bulletin (article) \\
\hline File Information & pre498.pdf \\
\hline
\end{tabular}

Instructions for use 


\section{On the motion by singular interfacial energy}

Yoshikazu Giga, Maurizio Paolini and Piotr Rybka

Series \# 498. November 2000 


\section{HOKKAIDO UNIVERSITY PREPRINT SERIES IN MATHEMATICS}

\#474 T. Nakazi, Norm inequalities for some singular integral operators, 13 pages. 1999.

\#475 A. Inoue and Y. Kasahara, Asymptotics for prediction errors of stationary processes with reflection positivity, 15 pages. 1999.

\#476 T. Sano, On affine parallels of generic plane curves, 8 pages. 1999.

\#477 T. Nakazi, On an invariant subspace whose common zero set is the zeros of some function, 11 pages. 1999.

\#478 M.-H. Giga and Y. Giga, Generalized motion by nonlocal curvature in the plane, 68 pages. 2000.

\#479 M.-H. Giga and Y. Giga, Crystalline and level set flow - Convergence of a crystalline algorithm for a general anisotropic curvature flow in the plane, 16 pages. 2000 .

\#480 A. Arai and M. Hirokawa, Stability of ground states in sectors and its application to the Wigner-Weisskopf model, 16 pages. 2000 .

\#481 T. Nakazi, Two dimensional $Q$-algebras, 11 pages. 2000.

\#482 N. H. Bingham and A. Inoue, Tauberian and Mercerian theorems for systems of kernels, 16 pages. 2000.

\#483 N. H. Bingham and A. Inoue, Abelian, Tauberian and Mercerian theorems for arithmetic sums, 29 pages. 2000.

\#484 I. A. Bogaevski and G. Ishikawa, Lagrange mappings of the first open Whitney umbrella, 22 pages. 2000.

\#485 A. Arai and H. Kawano, A class of deformations of the Schrödinger representation of the Heisenberg commutation relation and exact solution to a Heisenberg equation and a Schrödinger equation, 22 pages. 2000 .

\#486 T. Nakazi, Functions in $N_{+}$with the positive real parts on the boundary, 21 pages. 2000.

\#487 Y. Shibukawa, Classification of the $R$-operator, 36 pages. 2000.

\#488 A. Inoue, Asymptotic behaviour for partial autocorrelation functions of fractional ARIMA processes, 20 pages. 2000 .

\#489 S. Ohtani, Construction of unramified Galois extensions over maximal abelian extensions of algebraic number fields, 14 pages. 2000.

\#490 T. Nakazi and T. Yamamoto, The real part of an outer function and a Helson-Szegö weight, 13 pages. 2000 .

\#491 A. Yamagami, On Gouvêás conjecture on controlling the conductor, 11 pages. 2000.

\#492 I. Tsuda and M. Hatakeyama, Making sense of internal logic: Theory and a case study, 10 pages. 2000.

\#493 I. Tsuda, Towards an interpretation of dynamic neural activity in terms of chaotic dynamical systems, 73 pages. 2000.

\#494 T. Mikami, Optimal control for absolutely continuous stochastic processes and the mass transportation problem, 17 pages. 2000 .

\#495 M. Arisawa and Y. Giga, Anisotropic curvature flow in a very thin domain, 21 pages. 2000.

\#496 T. Nakazi, Backward shift invariant subspaces in the bidisc, 9 pages. 2000.

\#497 Y. Giga, K. Inui, J. Kato and S. Matsui, Remarks on the uniqueness of bounded solutions of the NavierStokes equations, 4 pages. 2000. 


\title{
On the motion by singular interfacial energy*
}

\author{
Yoshikazu Giga, Maurizio Paolini and Piotr Rybka \\ Department of Mathematics, Hokkaido University, Sapporo 060-0810, Japan \\ Dipartimento di Matematica e Fisica, Università Cattolica del Sacro Cuore, \\ via Trieste 17, 25121 Brescia, Italy \\ Institute of Applied Mathematics and Mechanics, Warsaw University, \\ ul. Banacha 2, 07-097 Warsaw, Poland
}

\begin{abstract}
Anisotropic curvature flow equations with singular interfacial energy are important for good understanding of motion of phase-boundaries. If the energy and the interfacial surface were smooth, then the speed of the interface would be equal to the gradient of the energy. However, this is not so simple in the case of non-smooth crystalline energy. But it's well-known that a unique gradient characterization of the velocity is possible if the interface is a curve in the two-dimensional space.

In this paper we propose a notion of solution in the three-dimensional space by introducing geometric subdifferentials and characterizing the speed. We also give a counterexample to a problem concerning the Cahn-Hoffman vector field on a facet, a flat portion of the interface.
\end{abstract}

Key words: . singular energies, geometric subdifferentials, canonical restrictions, facets, curvature flow

\section{Introduction}

Anisotropic curvature flow equations with singular interfacial energy were proposed independently by J. Taylor [38] and by S. Angenent and M. Gurtin [3] more than a decade ago to model motion of phase-boundaries in material sciences and the theory of crystal growth. A typical example takes the form

$$
\beta(\mathbf{n}) V=-\operatorname{div}_{\Gamma_{t}}[(\nabla \gamma)(\mathbf{n})] \text { on } \Gamma_{t} .
$$

\footnotetext{
*In the memory of Professor Masaya Yamaguti
} 
Here $V$ denotes the normal velocity of an interface $\Gamma_{t}$ in $\mathbf{R}^{n}$ in the direction of a unit outer normal $\mathbf{n}$ of $\Gamma_{t} ; \beta$ is a kinetic coefficient and it is assumed to be positive. The function $\dot{\gamma}: \mathbf{R}^{n} \rightarrow[0, \infty)$ is an interfacial energy density and it is assumed to be positively homogeneous of degree one; $\nabla \gamma$ denotes the gradient of $\gamma$ in $\mathbf{R}^{n}$ and $\operatorname{div}_{\Gamma_{t}}$ denotes the surface divergence. If $\gamma(p)=|p|$ and $\beta \equiv 1$, then (1.1) is nothing but the famous mean curvature flow equation. The problem (1.1) is (possibly degenerate) parabolic provided that $\beta>0$ and $\gamma$ is convex. An extreme but important example of singular energy $\gamma$ is the crystalline energy, i.e. Frank $\gamma=\left\{p \in \mathbf{R}^{n} ; \gamma(p) \leq 1\right\}$ is a convex (bounded) polyhedron in $\mathbf{R}^{n}$. In this case the meaning of (1.1) is not at all clear even if we assume that $\Gamma_{t}$ is smooth since the smoothing effect is so strong so that it is expected to be nonlocal.

When $n=2$ so that $\Gamma_{t}$ is a curve in the plane, the problem (1.1) for singular energy is by now well-studied. Three different notions of solutions have been proposed when $\gamma$ is crystalline.

(i) Crystalline flow. A special class of polygonal flows is considered and (1.1) is interpreted as a system of ordinary differential equations. A key assumption is that the speed of each segment is a constant (depending on time) on the segment. This flow is proposed by [38], [3]; see also [39]. Several properties of solutions are well-studied [38], [40], [36], [37], [22]. The theory still works for the more general equation

$$
\beta(\mathbf{n}) V=-\operatorname{div}_{\Gamma_{t}}[(\nabla \gamma)(\mathbf{n})]+C
$$

with some constant $C$ or even for

$$
V=f\left(\mathbf{n},-\operatorname{div}_{\Gamma_{t}}[(\nabla \gamma)(\mathbf{n})]\right)
$$

for a large class of coupling functions $f[3],[40],[19]$. The Stefan type problem with crystalline interfacial energy has been studied in the papers [31], [32], [33]. Thus, sometimes it is natural to admit nonconstant $C$ (cf. [16], [18]).

(ii) Variational flow. This is proposed by [12] and its properties are studied in [11] when $\Gamma_{t}$ is the graph of a function $u$. The idea is to interpret (1.1) by a variational inequality $\partial_{t} u \in-\partial \varphi(u)$ with some choice of the energy $\varphi$. Unfortunately, this approach is so far limited for graph curves i.e. graphs of functions and, moreover, no driving force term $C$ in (1.2) is allowed. However, solutions can be constructed for a very large class of initial data. We refer [30], [21] for a review of the theory.

(iii) Viscosity flow. This is developed by [15], [17] for graph curves and [20] for general closed curves. The idea is based on comparison principle. In [15], [17], [20] 
the theory of viscosity solutions is extended to nonlocal smoothing (diffusion) effects. This theory covers the general equation (1.3) with general singular interfacial energy. Another merit is that the problem with smooth energy $\gamma$ and crystalline $\gamma$ can be treated in the same framework so it is useful in understanding approximability of solutions [19].

For consistency of these notions we refer to [12], [11], [14], [19]. Since crystalline flow is characterized by ODEs, it provides an algorithm to calculate numerically motion by smooth energy. The convergence of the algorithm has been proved in various settings [12], [26], [27], [29], [17], [18], [19]. Approximation of crystalline flow with motion by smooth energy has been also established [12], [17], [18], [19]. Convergence of time semi-discretized variational solution is also established [2]. The Allen-Cahn type approximation is also discussed in [4], [5], [42], [24].

For surface evolution in $\mathbf{R}^{3}$ less is known. In [23] a crystalline flow is proposed and a uniform speed on a facet (flat portion) is characterized by a canonical restriction of the subdifferential of the interface energy. Unfortunately, their calculation of the canonical restriction is not correct [23, Lemma 9.6]. Later in [6] it is shown that the constancy of velocity on a facet may not hold if the flow fulfills the comparison principle. A facet may break. However, still a reasonable choice of the speed seems to be given by a canonical restriction of the subdifferentials as in [23].

In this paper we shall prove this conjecture by defining geometric subdifferentials of interfacial energy and strong solutions to (1.1). We shall prove that the speed must be equal to the canonical restriction (minimal element in $L^{2}$ norm) of geometric subdifferentials. However, we are unable to prove the existence of our solutions nor we are able to handle topology changes. The comparison principle is likely to hold but we do not pursue this problem.

Bellettini and Novaga [5] give a notion of solution satisfying the comparison principle (but they do not show existence of solutions nor characterize the speed). Our definition is not comparable to their definition. At this point we should explain that the speed is expected to be characterized by the minus divergence of a suitable Cahn-Hoffman vector field. Bellettini and Novaga [7] assume existence of a bounded Cahn-Hoffman vector field with bounded surface divergence while we require that the divergence is in $L^{2}$. For Lipschitz surfaces studied in [7] the authors assumed the existence of a Lipschitz Cahn-Hoffman vector field. However, such an assumption is often too strong since there is a minimal crystalline surface whose Cahn-Hoffman vector field [9], [28], [23] is not continuous [10]. Our definition does not require the existence of continuous Cahn-Hoffman vector field. 
Some time ago Cahn and Hoffman [9] formulated a conjecture saying that a reasonable Cahn-Hoffman vector field whose minus divergence equals the speed can be written as a gradient of a function on a facet. Unfortunately, this conjecture is false in general; we shall give a counterexample.

This paper is organized as follows. In $\S 2$ we introduce a good coordinate system to define geometric subdifferentials of the interfacial energy. In $\S 3$ we define geometric subdifferentials and their characterization. In $\S 4$ we prove that the velocity of our flow agrees with the canonical restriction of the interfacial energy. In $\S 5$ we disprove a conjecture of Cahn and Hoffman [9].

\section{A class of surfaces}

The goal of this section is to give a notion of admissible coordinate systems of the surface so that "geometric subdifferential" of interfacial energy is defined. Let $\gamma$ be a Lipschitz continuous real-valued convex function on $\mathbf{R}^{3}$. Assume that $\gamma$ is positively homogeneous of degree one, i.e., $\gamma(\lambda p)=\lambda \gamma(p)$ for all $\lambda>0$ and $p \in \mathbf{R}^{3}$. Assume that $\gamma(p)>0$ for $p \neq 0$. The set

$$
\left\{p \in \mathbf{R}^{3} ; \gamma(p) \leq 1\right\}
$$

is called the Frank diagram of $\gamma$ and is denoted by Frank $\gamma$. By our assumption Frank $\gamma$ is a nonempty, bounded convex closed set. We shall distinguish vertices, edges of Frank $\gamma$. Let $\partial \gamma(p)$ denote the subdifferential of $\gamma$ at $p$, i.e.,

$$
\partial \gamma(p)=\left\{w \in \mathbf{R}^{3} ; \gamma(p+q)-\gamma(p) \geq w \cdot q \text { for all } q \in \mathbf{R}^{3}\right\},
$$

where $w \cdot q$ is the standard inner product of $w, q \in \mathbf{R}^{3}$. The set $\partial \gamma(p)$ is a closed convex set in $\mathbf{R}^{3}$. For $p \neq 0$ if $\partial \gamma(p)$ is not a singleton, it is either a line segment or a planar convex set not contained in a line. For $p$ on the boundary $\partial$ (Frank $\gamma$ ) of Frank $\gamma$, we say that $p$ is a vertex (of Frank $\gamma$ ) if $\partial \gamma(p)$ is a planar convex set (not contained in a line). If $\partial \gamma(p)$ is a line segment, we say that $p \in \partial$ (Frank $\gamma$ ) is an edge point (of Frank $\gamma$ ). In addition we assume that $\gamma$ is of class $C^{1,1}$ away from 0 , vertices and edge points of the Frank diagram.

These notions are consistent with our intuition. At this point let us remind that $\partial \gamma(0)$ is often called the Wulff shape of $\gamma$ and is denoted Wulff $\gamma$. It is the convex conjugate of Frank $\gamma$.

We consider an oriented Lipschitz surface $S$ embedded in $\mathbf{R}^{3}$. Let $\mathbf{n}$ be the unit normal vector field which determines the orientation of $S$. A subset $F$ of $S$ is a facet 
if it is regarded as the closure of a connected component of a nonempty open set in some plane (support plane) and it is maximal among those having this property. A subset $\ell$ of $S$ is a line segment if it is regarded as maximal nontrivial closed interval on a line (support line). A subset $L$ of $S$ is a directionally linear surface if it is a union of a continuous family of disjoint line segments and it contains no interior points of any facet in a plane. Let $\mathcal{O}_{i}(1 \leq i \leq m)$ be a bounded cylindrical domain in the sense that there exists a rotation $R_{i} \in S O(3)$, a bounded domain $\Omega_{i}$ with Lipschitz boundary in $\mathbf{R}^{2}$ and a bounded open interval $I_{i}$ such that $\mathcal{O}_{i}=R_{i}\left(\Omega_{i} \times I_{i}\right)$.

Definition 2.1 ( $\gamma$-facet and $\gamma$-directionally linear surface).

(i) Let $F$ be a facet in $S$. If the orientation of $F$ is in the direction of a vertex of Frank $\gamma$, we call $F$ a $\gamma$-facet.

(ii) Let $L$ be a directionally linear surface in $S$ with orientation $\mathbf{n}$. If the orientation $\mathbf{n}$ exists almost everywhere on a line segment $\ell$ forming $L$ and $\mathbf{n}$ is in the direction of a point on an edge of Frank $\gamma$ (independent of $\ell$ ), we call $L$ a $\gamma$-directionally linear surface.

(iii) Let $\Sigma \subset S$ be the union of $\gamma$-facets and $\gamma$-directionally linear surfaces. We say that a point $P$ of $S$ is a localizable point of $S$ if $P$ does not belong to $\Sigma$. The set of all localizable points is denoted by $\Sigma^{c}(=S \backslash \Sigma)$.

Definition 2.2. We say that a covering $\mathcal{A}=\left\{\mathcal{O}_{i}\right\}_{i=1}^{m}$ of $S$ with bounded cylindrical domains is an admissible coordinate system of $S$ with respect to $\gamma$ if the following properties are fulfilled.

(i) The set $R_{i}^{-1}\left(S \cap \mathcal{O}_{i}\right)$ is represented as the graph of a Lipschitz function on $\Omega_{i}$. The orientation $R_{i}^{-1} \mathbf{n}$ is taken upward for all $i=1, \ldots, m$. Here $\Omega_{i}$ and $R_{i}$ are as in the definition of cylindrical domains.

(ii) A $\gamma$-facet $F$ is contained in $\mathcal{O}_{j}$ for some $j=1, \ldots, m$.

(iii) A line segment $\ell$ forming a $\gamma$-directionally linear surface is contained in $\mathcal{O}_{j}$ for some $j=1, \ldots, m$.

(iv) The orientation $\mathbf{n}(P)$ does not point to neither a vertex nor an edge point of Frank $\gamma$ for $\mathcal{H}^{2}$-almost all $P \in \Sigma^{c}$; here $\mathcal{H}^{2}$ denotes the two-dimensional Hausdorff measure.

Example 2.3. Assume that Frank $\gamma$ is a convex polyhedron. Such a $\gamma$ is called a crystalline energy density. If $p \cdot q>0$ for every pair of adjacent vertices $p, q$ of Frank $\gamma$, we say that $\mathcal{N}=\{p /|p|, p$ is a vertex of Frank $\gamma\}$ is acute. If $\mathcal{N}$ is acute, it is easy to see that the Wulff shape of $\gamma$ has an admissible coordinate system (cf. [23]) 
In general Wulff $\gamma$ may not have an admissible coordinate system. For example, if Frank $\gamma$ is regular octahedron (so that $\mathcal{N}$ is not acute), the Wulff shape which is a cube has no admissible coordinate systems. We thus observe that there exist Lipschitz surfaces which have no admissible coordinate systems.

Definition 2.4. An oriented Lipschitz embedded surface in $\mathbf{R}^{3}$ is called $\mathcal{A}$-covered if there is an admissible coordinate system $\mathcal{A}$.

\section{Geometric subdifferentials}

Let $S$ be an oriented Lipschitz surface in $\mathbf{R}^{3}$ with orientation $\mathbf{n}$. Let $I(S)$ be the interfacial energy with density $\gamma$, i.e.,

$$
I(S)=\int_{S} \gamma(\mathbf{n}) d \mathcal{H}^{2},
$$

where $\mathcal{H}^{2}$ denotes the surface element. Our goal in this section is to define the 'geometric subdifferential' of $I$.

Assume that $S$ is $\mathcal{A}$-covered so that it has an admissible coordinate system $\mathcal{A}=\left\{\mathcal{O}_{i}\right\}_{i=1}^{m}$. For each $i=1, \ldots, m$, the set $S \cap \mathcal{O}_{i}$ is represented as

$$
\Sigma_{i}:=R_{i}^{-1}\left(S \cap \mathcal{O}_{i}\right)=\left\{x_{3}=f_{i}\left(x_{1}, x_{2}\right),\left(x_{1}, x_{2}\right) \in \Omega_{i}\right\}
$$

with some Lipschitz function $f_{i}$ on $\Omega_{i}$. By rotation we observe that

$$
I\left(S \cap \mathcal{O}_{i}\right)=\int_{S \cap \mathcal{O}_{i}} \gamma(\mathbf{n}) d \mathcal{H}^{2}=\int_{\Sigma_{i}} \gamma_{i}(\mathbf{n}) d \mathcal{H}^{2}, \gamma_{i}(p)=\gamma\left(R_{i} p\right),
$$

where $\mathbf{n}$ in the second integral denotes that orientation of $\Sigma_{i}$ inherited from $S$. Since $\mathbf{n}=\left(-\nabla f_{i}, 1\right) / \sqrt{1+\left|\nabla f_{i}\right|^{2}}, d \mathcal{H}^{2}=\sqrt{1+\left|\nabla f_{i}\right|^{2}} d x$ and $\gamma_{i}$ is positively homogeneous of degree one, $I\left(S \cap \mathcal{O}_{i}\right)$ is now represented as

$$
I\left(S \cap \mathcal{O}_{i}\right)=\int_{\Omega_{i}} \gamma_{i}\left(-\nabla f_{i}, 1\right) d x
$$

where $\nabla f_{i}=\left(\partial f_{i} / \partial x_{1}, \partial f_{i} / \partial x_{2}\right)$. When we apply $R_{i}$ or $R_{i}^{-1}$ as a matrix to a vector $\mathbf{v}$ in $\mathbf{R}^{n}$, when $\mathbf{v}$ is interpreted as a column vector. To define subdifferentials we fix a function space and a functional which is convex and lower semicontinuous on this function space. For this purpose we modify $\gamma_{i}$. Let $K_{i}$ be strictly larger than the Lipschitz constant of $f_{i}$. For $\gamma_{i}$ we define a convex function $W_{i}(q)$ on $\mathbf{R}^{2}$ which is coercive, i.e., $\lim _{q \rightarrow \infty} W(q) /|q|=\infty$, and satisfies

$$
W_{i}(q)=\gamma_{i}(-q, 1) \text { for }|q| \leq K_{i} .
$$


Let $H$ be the real Hilbert space $L^{2}\left(\Omega_{i}\right)$ equipped with the inner product $\langle u, v\rangle=$ $\int_{\Omega_{i}} u v d x$. For $u \in H$ we define $\varphi_{i}: H \rightarrow(-\infty, \infty]$ as

$$
\varphi_{i}(u)= \begin{cases}\int_{\Omega_{i}} W_{i}(\nabla u) d x, & \text { if } \nabla u \in L^{1}\left(\Omega_{i}\right) \text { and } W_{i}(\nabla u) \in L^{1}\left(\Omega_{i}\right) \\ +\infty, & \text { with } u=f_{i} \text { on } \partial \Omega_{i}, \\ +\infty \text { otherwise. }\end{cases}
$$

Since $W_{i}$ is convex and coercive, it is well-known that $\varphi_{i}$ is a lower semicontinuous, convex function on $H$ and $\varphi_{i} \not \equiv \infty$; see e.g. [23, Lemma 9.1]. Thus the set-valued function

$$
u \mapsto \partial \varphi_{i}(u)
$$

is a maximal monotone operator, where $\partial \varphi_{i}(u)$ denotes the subdifferential of $\varphi_{i}$ at $u$, i.e.

$$
\partial \varphi_{i}(u)=\left\{w \in H ; \varphi_{i}(u+h)-\varphi_{i}(u) \geq\langle w, h\rangle \text { for all } h \in H\right\} .
$$

This set may be empty, but it must be convex and closed. We are now in a position to define the geometric subdifferential $\partial I(S)$ at $S$ as a subset of the Hilbert space $L^{2}(S)$ equipped with the standard inner product $\langle w, v\rangle=\int_{S} w v d \mathcal{H}^{2}$ for $w, v \in L^{2}(S)$.

Definition 3.1. A function $w \in L^{2}(S)$ is said to belong to the geometric subdifferential of $I$ at $S$ if for almost every $P \in S$ and for all $i \in\{1, \ldots, m\}$ such that $P \in \mathcal{O}_{i}$ the identity $w(P)=\tilde{w}\left(x^{\prime}\right)$ holds for some $\tilde{w} \in \partial \varphi_{i}\left(f_{i}\right)$, where $x^{\prime}=\pi\left(R_{i}^{-1} P\right)$ provided that one of the following conditions is fulfilled.

(i) $P \in \mathcal{O}_{i}$ is contained in a $\gamma$-facet $F(\subset S)$ contained in $\mathcal{O}_{i}$.

(ii) $P \in \mathcal{O}_{i}$ is contained in a $\gamma$-directionally linear surface $L(\subset S)$ contained in $\mathcal{O}_{i}$.

(iii) $P \in \mathcal{O}_{i}$ is a localizable point of $S$.

Here $\pi$ denotes the orthogonal projection $\Omega_{i} \times I_{i} \rightarrow \Omega_{i}$. The geometric subdifferential of $I$ at $S$ is denoted $\partial I(S)$.

The set $\partial I(S)$ may be empty, but it must be convex and closed since so are $\partial \varphi_{i}$ (as pointed out in [12] the set $\partial \varphi_{i}$ can be empty). Thus unless $\partial I$ is empty, there exists a unique $w \in \partial I(S)$ that minimizes the $L^{2}$-norm $\|\bar{w}\|$ of $\bar{w} \in \partial I(S)$. We call $w$ a canonical restriction (or minimal section) of $\partial I(S)$ and it will be denoted by $\partial^{0} I(S)$. Although our geometric subdifferential is not exactly a subdifferential, it has similar properties. If $S$ is smooth and $S_{h}$ is a surface moved by $h$ in the direction of $\mathbf{n}$, then

$$
I\left(S_{h}\right)-I(S) \geq \int_{S} h w d \mathcal{H}^{2}
$$


for $w \in \partial I(S)$ if the absolute value $|h|$ is small. Unless $\gamma$ is smooth, such a setting is not realistic. However, this indicates that our subdifferential formally measures variation of $I(S)$ with respect to normal direction.

We shall prove that the value of $w \in \partial I(S)$ at a localizable point is independent of the choice of $w \in \partial I(S)$, provided that $\Sigma^{c}$ enjoys some additional smoothness. But first, we shall establish a local representation of $\partial I(S)$.

Theorem 3.2. Assume that $S$ is $\mathcal{A}$-covered. Let $\Sigma^{c}$ denote the set of all localizable points of $S$. Assume that $\Sigma^{c}$ is open. If $w_{1}, w_{2} \in \partial I\left(S \cap \mathcal{O}_{i}\right)$, for some $i$, then $w_{1}(P)=w_{2}(P)$ for $\left(\mathcal{H}^{2}\right.$-a.e. $) P$ in $\Sigma^{c} \cap \mathcal{O}_{i}$.

Such a statement does not preclude possibility that values of $w \in \partial I(S)$ may depend upon particular chart $\mathcal{O}_{i}$. Later, we shall show that this does not happen if we know some further smoothness of $\Sigma^{c}$.

To prove Theorem 3.2 we recall a characterization of the subdifferential $\partial \varphi_{i}$ (for each $i=1, \ldots, m$ ) which is due to Attouch and Damlamian [1].

Lemma 3.3. Assume that $u \in L^{2}\left(\Omega_{i}\right)$. A function $w \in L^{2}\left(\Omega_{i}\right)$ belongs to $\partial \varphi_{i}(u)$ if and only if there is a bounded measurable function $\eta$ on $\Omega_{i}$ such that

$$
w=-\operatorname{div} \eta \text { in } \Omega_{i}
$$

in the distribution sense and $\eta(x) \in \partial W_{i}(\nabla u(x))$ for almost every $x \in \Omega_{i}$.

Proof of Theorem 3.2. By definition of $W_{i}$ we easily observe that

$$
\partial W_{i}(q)=-\pi\left(R_{i}^{-1}(\partial \gamma)\left(R_{i} P\right)\right), P=(-q, 1) /\left(|q|^{2}+1\right)^{1 / 2} \text { for } q \in \mathbf{R}^{2} .
$$

By our assumption (Definition 2.1 (iv)) for $\mathcal{H}^{2}$-a.e. $P \in \Sigma^{c}$ the set $\partial \gamma(\mathbf{n}(P))$ is a singleton. By (3.1) this implies that $\partial W_{i}\left(\nabla f_{i}\left(x^{\prime}\right)\right)$ for $x^{\prime}=\pi\left(R_{i}^{-1} P\right)$ is a singleton for $\mathcal{H}^{2}$-a.e. $P \in \Sigma^{c} \cap \mathcal{O}_{i}$.

Assume that $w_{1}, w_{2} \in \partial I\left(S \cap \mathcal{O}_{i}\right)$. For $\mathcal{H}^{2}$-a.e. $P \in S \cap \mathcal{O}_{i}, w_{j}(P)=\tilde{w}_{j}\left(x^{\prime}\right)(j=$ $1,2)$ and $\tilde{w}_{j} \in \partial \varphi_{i}\left(f_{i}\right)$. By Lemma 3.3 with $u=f_{i}$.

$$
\tilde{u}_{j}=-\operatorname{div} \eta_{j}, \eta_{j}\left(x^{\prime}\right) \in \partial W_{i}\left(\nabla f_{i}\left(x^{\prime}\right)\right), \quad(j=1,2)
$$

Since we have assumed that $\Sigma^{c}$ is open, the set $D_{i}=\pi\left(R_{i}^{-1}\left(\Sigma^{c} \cap \mathcal{O}_{i}\right)\right)$ is open in $\Omega_{i}$. Since $\partial W_{i}\left(\nabla f_{i}\left(x^{\prime}\right)\right)$ is a singleton in $D_{i}$, then due to $(3.2) \tilde{\eta_{1}}=\tilde{\eta}_{2}$ holds on $D_{i}$ a.e. We have thus proved that $\tilde{w}_{1}=\tilde{w}_{2}, \mathcal{H}^{2}$-a.e. in $D_{i}$. The proof is now complete. 
Remark 3.4. By the representation of subdifferential $\partial \varphi_{i}$ in Lemma 3.3 it is clear that $\partial I(S)$ does not depend on the choice of the extension of $W_{i}$ to $\mathbf{R}^{2}$.

If we have regularity of $\nabla \gamma(\mathbf{n}(P))$ on $\Sigma^{c}$, then we have uniqueness of representation of $w \in \partial I(S)$ and also the value $w$ on $\Sigma^{c}$ is computable. We do not attempt to prove optimal results, but we establish some necessary basic ones.

Lemma 3.5. Assume that $S$ is $\mathcal{A}$-covered and that $\left\{\mathcal{O}_{i}\right\}_{i=1}^{m}$ is the $\mathcal{A}$-covering. We set $U_{i}=f_{i}^{-1}\left(R_{i}^{-1}\left(\Sigma^{c} \cap \mathcal{O}_{i}\right)\right)$ and we assume that $\left.f_{i}\right|_{U_{i}}$ is a $C^{1,1}$ function for each $i=1,2, \ldots, m$. Then,

(i) the vector field $\xi(P)=\nabla \gamma(\mathbf{n}(P))$ is Lipschitz continuous in $P \in \Sigma^{c}$ and so is $P \mapsto \mathbf{n}(P)$.

(ii) There exists $\overline{\mathbf{n}}$ an extension of the vector field $\mathbf{n}(P)$ to a neighborhood of $\Sigma^{c}$ in $\mathbf{R}^{3}$, which is $\mathcal{H}^{2}$-a.e. differentiable.

Proof. (i) Smoothness of $\Sigma^{c}$ makes the claim obvious.

(ii) If $R_{i}^{-1} y$ belongs to $\Omega_{i} \times I_{i}$, then we set

$$
\overline{\mathbf{n}}(y)=R_{i}\left(-\nabla f_{i}(x), 1\right) /\left(1+\left|\nabla f_{i}(x)\right|^{2}\right)^{1 / 2}
$$

where $x=\pi R_{i}^{-1} y$. It is now clear that $\overline{\mathbf{n}}$ and $\xi$ have the desired properties.

This Lemma provides us with sufficiently differentiable extensions of $\mathbf{n}$. As we shall see, they play an important role.

Theorem 3.6. Let us suppose that the assumptions of previous Lemma hold. If $\overline{\mathbf{n}}$ is an arbitrary $\mathcal{H}^{2}$-a.e. differentiable extension of vector field $\mathbf{n}$, then

$$
\operatorname{div}_{S} \xi=\operatorname{div}_{\mathbf{R}^{3}} \nabla \gamma(\overline{\mathbf{n}})
$$

holds $\mathcal{H}^{2}$-a.e. on $\Sigma^{c}$, where $\operatorname{div}_{S}$ denotes the surface divergence. Moreover, if $w_{k} \in$ $\partial I(S), k=1,2$, then $w_{1}(P)=w_{2}(P)$ for $\left(\mathcal{H}^{2}\right.$-a.e. $) P$ in $\Sigma^{c}$.

Remark 3.7. (i) (Surface divergence) For a Lipschitz vector field $\zeta$ on an open subset $M$ of $S$ one is able to define surface divergence as

$$
\left(\operatorname{div}_{S} \zeta\right)\left(P^{\prime}\right)=\sum_{i=1}^{2}\left(D_{\tau_{i}} \zeta\right)(P) \cdot \tau_{i}
$$


where $\left\{\tau_{1}, \tau_{2}\right\}$ is an orthogonal basis of the tangent space $T_{P} S$ at $P$ and $D_{\tau_{i}}$ is the directional derivative in the direction of $\tau_{i}$ (cf. [35]). This quantity is well-defined for $\mathcal{H}^{2}$-almost all $P \in M$. Since

$$
\left(\operatorname{div}_{S} \zeta\right)(P)=\operatorname{trace}((I-\mathbf{n}(P) \otimes \mathbf{n}(P)) \nabla \zeta)
$$

for any Lipschitz extension of $\zeta$ to a neighborhood of $U$ in $\mathbf{R}^{3}$, in particular, $\operatorname{div}_{S} \zeta$ is independent of the way of extension. Unfortunately, if $\zeta$ is not Lipschitz, the above definition loses its meaning for Lipschitz surfaces.

(ii) From Theorem 3.6 it follows that the value of $w \in \partial I(S)$ on $\Sigma^{c}$ is independent of the choice of admissible coordinate system.

Proof of Theorem 3.6. Let us consider $\overline{\mathbf{n}}$ an arbitrary $\mathcal{H}^{2}$-a.e. differentiable extension of $\mathbf{n}$. By an equivalent definition of surface divergence, we see

$$
\operatorname{div}_{S}(\nabla \gamma(\mathbf{n}))=\operatorname{div}_{S}(\nabla \gamma(\overline{\mathbf{n}}))=\operatorname{div}(\nabla \gamma(\overline{\mathbf{n}}))-\overline{\mathbf{n}} \cdot D_{\overline{\mathbf{n}}}(\nabla \gamma(\overline{\mathbf{n}}))
$$

By our assumptions we are allowed to calculate

$$
\overline{\mathbf{n}} \cdot D_{\overline{\mathbf{n}}}(\nabla \gamma(\overline{\mathbf{n}}))=D_{\overline{\mathbf{n}}}(\overline{\mathbf{n}} \cdot \nabla \gamma(\overline{\mathbf{n}}))-D_{\overline{\mathbf{n}}} \overline{\mathbf{n}} \cdot \nabla \gamma(\overline{\mathbf{n}}) \quad \text { for } \mathcal{H}^{2} \text {-a.e. near } P .
$$

By the homogeneity we see $\overline{\mathbf{n}} \cdot \nabla \gamma(\overline{\mathbf{n}})=\gamma(\overline{\mathbf{n}})$ so that

$$
\overline{\mathbf{n}} \cdot D_{\overline{\mathbf{n}}}(\nabla \gamma(\overline{\mathbf{n}}))=0
$$

We thus obtain that

$$
\operatorname{div}_{S}(\nabla \gamma(\mathbf{n}))=\operatorname{div}_{\mathbf{R}^{3}}(\nabla \gamma(\overline{\mathbf{n}})),
$$

and this result does not depend upon particular extension of $\mathbf{n}$. Let us now assume that $w_{1}, w_{2} \in \partial I(S)$. We have already established in Theorem 3.2 that if we fix a chart $\mathcal{O}_{i}$, then $\partial I\left(S \cap \mathcal{O}_{i}\right)$ is uniquely defined. Suppose then that $w_{k} \in \partial I\left(S \cap \mathcal{O}_{i_{k}}\right)$, $k=1,2$ and $\mathcal{O}_{i_{1}} \cap \mathcal{O}_{i_{2}} \neq \emptyset$. As we know,

$$
\operatorname{div}_{S} \xi=\operatorname{div}_{\mathbf{R}^{3}}(\nabla \gamma(\overline{\mathbf{n}}(P))
$$

and we can take a convenient extension $\overline{\mathbf{n}}$. In $\mathcal{O}_{i_{k}}, k=1,2$ we use the extension defined in the course of proof of Lemma 3.5. Thus,

$$
w_{k}=-\operatorname{div}_{x^{\prime}}\left[\nabla W_{i_{k}}\left(\nabla f_{i_{k}}\left(x^{\prime}\right)\right)\right]=\operatorname{div}_{\mathbf{R}^{3}}(\nabla \gamma(\overline{\mathbf{n}}(P)), \quad k=1,2
$$

for $\mathcal{H}^{2}$ - a.e. $P$ in $\mathcal{O}_{i_{1}} \cap \mathcal{O}_{i_{2}}$. Our Theorem follows. 
For a given $\eta$ in Lemma 3.3 with $u=f_{i}, \eta\left(x^{\prime}\right) \in \partial W_{i}\left(\nabla f_{i}\left(x^{\prime}\right)\right)$ we wonder whether there is a $\xi$ in $\mathcal{O}_{i}$ (not only on $S \cap \mathcal{O}_{i}$ ) such that

$$
\begin{gathered}
\operatorname{div}_{\mathbf{R}^{2}} \eta\left(x^{\prime}\right)=-\operatorname{div}_{\mathbf{R}^{3}} \xi(P), \quad x^{\prime}=\pi R_{i}^{-1} P \\
\xi(P) \in \partial \gamma(\mathbf{n}(P)) .
\end{gathered}
$$

Indeed, such a $\xi$ exists, thus the so called Cahn-Hoffman vector field can be constructed in one chart. In fact, the homogeneity of $\gamma$ yields

$$
(\partial \gamma)(\tilde{P}) \cdot \tilde{P}=\gamma(\tilde{P}) \text { with } R_{i} P=\tilde{P}
$$

By (3.1) for each $\eta_{0} \in \partial W(q)$, there is a unique $\xi_{0} \in \partial \gamma\left(R_{i} P\right)$ that satisfies $\eta_{0}=$ $-\pi\left(R_{i}^{-1} \xi_{0}\right)$; the mapping $\eta_{0} \mapsto \xi_{0}$ is denoted by $\Xi$. Using this mapping, we obtain that

$$
\operatorname{div} \eta\left(x^{\prime}\right)=-\operatorname{div}_{x^{\prime}} \pi\left(R_{i}^{-1} \Xi(\eta)\right)\left(x^{\prime}\right)
$$

with $\Xi(\eta)\left(x^{\prime}\right) \in \partial \gamma(\mathbf{n}(P)), x^{\prime}=\pi R_{i}^{-1} P$. We set $\xi(P)$ for $P \in \mathcal{O}_{i}$ by

$$
\xi(P)=\Xi(\eta)\left(\pi R_{i}^{-1} P\right) .
$$

Since

$$
\begin{aligned}
-\operatorname{div}_{x^{\prime}} \pi\left(R_{i}^{-1} \Xi(\eta)\right)\left(x^{\prime}\right) & =-\operatorname{div}_{x^{\prime}} R_{i}^{-1} \Xi(\eta)\left(x^{\prime}\right) \\
& =-\operatorname{div}_{\mathbf{R}^{3}} \xi(P),
\end{aligned}
$$

the vector field $\xi$ fulfills (3.3), (3.4).

In general it is not clear that $\operatorname{div}_{S} \xi$ is well-defined for $\xi$ defined by (3.6). However, on $\gamma$-facet or some $\gamma$-directionally flat surface we have the expected results.

Lemma 3.8. Let $\xi$ be defined by (3.6).

(i) Let $F$ be a $\gamma$-facet of $S$ in $\mathcal{O}_{i}$. Then

$$
\operatorname{div}_{S} \xi=\operatorname{trace}((I-\mathbf{n} \otimes \mathbf{n}) \nabla \xi)=\operatorname{div} \xi(P) \quad \text { for a.e. } P \in F
$$

where $\mathbf{n}$ is the orientation of $F$.

(ii) Let $L$ be a $\gamma$-directionally flat surface of $S$ in $\mathcal{O}_{i}$. Assume that its orientation $\mathbf{n}$ is orthogonal to one fixed vector $\tau$ (so that $\partial \gamma(\mathbf{n}) \cdot \nu$ is a singleton for $\nu=\tau \times \mathbf{n}$ ). Assume that the mapping $P \mapsto$ the element of $\partial \gamma(\mathbf{n}(P)) \cdot \nu$ (denoted $\nabla \gamma(\mathbf{n}) \cdot \nu)$ is Lipschitz continuous. Then

$$
\operatorname{div}_{S} \xi=\operatorname{div} \xi(P), \quad \text { for } \mathcal{H}^{2} \text {-a.e. } P \in F
$$


Proof. (i) By definition

$$
\operatorname{div}_{S} \xi(P)=\operatorname{div} \xi(P)-\mathbf{n} \cdot\left(D_{\mathbf{n}} \xi\right)(P)
$$

Since $\mathbf{n}(P)$ is constant on $F, \mathbf{n} \cdot\left(D_{\mathbf{n}} \xi\right)=D_{\mathbf{n}}(\xi \cdot \mathbf{n})$. Moreover, by $(3.5) \xi \cdot \mathbf{n}=\gamma(\mathbf{n})$. This quantity is constant on $F$ so $D_{\mathbf{n}}(\xi \cdot \mathbf{n})=0$.

(ii) We may assume, by rotation of coordinates of $\Omega_{i}$, that $\nu=\left(0, \nu_{2}, \nu_{3}\right)$ and $\mathbf{n}=\left(0, n_{2}, n_{3}\right)$ on $L$. Since $\partial \gamma(\mathbf{n}) \cdot \nu$ and $\partial \gamma(\mathbf{n}) \cdot \mathbf{n}=\{\gamma(\mathbf{n})\}$ are singletons,

$$
\left(\xi_{2}, \xi_{3}\right)=\left(\frac{\partial \gamma}{\partial p_{2}}(\mathbf{n}), \frac{\partial \gamma}{\partial p_{3}}(\mathbf{n})\right)=: \nabla^{\prime} \gamma(\mathbf{n})
$$

By (3.5) we now observe that

$$
\begin{aligned}
\mathbf{n}\left(D_{\mathbf{n}} \xi\right) & \left.=D_{\mathbf{n}}(\mathbf{n} \cdot \xi)-D_{\mathbf{n}} \mathbf{n} \cdot \xi=D_{\mathbf{n}} \gamma(\mathbf{n})-D_{\mathbf{n}} \mathbf{n}^{\prime} \cdot \nabla^{\prime} \gamma(\mathbf{n})\right) \\
& =D_{\mathbf{n}} \mathbf{n}^{\prime} \cdot \nabla^{\prime} \gamma(\mathbf{n})-D_{\mathbf{n}} \mathbf{n}^{\prime} \cdot \nabla^{\prime} \gamma(\mathbf{n})=0
\end{aligned}
$$

where $\mathbf{n}^{\prime}=\left(n_{2}, n_{3}\right)$. Thus $\operatorname{div}{ }_{S} \xi=\operatorname{div} \xi$ follows $\mathcal{H}^{2}$-almost everywhere in $L$.

Remark 3.9. The assumption that $\mathbf{n}$ is orthogonal to a single field vector $\tau$ is fulfilled if position vectors of an edge of Frank $\gamma$ belong to a plane containing the origin. We may relax this assumption saying that $\tau$ varies smoothly.

Remark 3.10. It is expected that $\partial I(S)$ is independent of the choice of admissible coordinate systems. Unfortunately, we are unable to prove this statement in this generality since we do not have a characterization of $\operatorname{div} \xi$ in (3.6) by a surface divergence. If $S$ consists of $\gamma$-facets up to $\mathcal{H}^{2}$-measure zero set (this case is called fully faceted), by Lemma 3.8 the quantity $\operatorname{div} \xi(P)$ is independent of the extension of $\xi$ and $W_{i}$, so $w \in \partial I(S)$ w.r.t. $\left\{\mathcal{O}_{i}\right\}$ also belongs to $\partial I(S)$ w.r.t. other admissible coordinates. See also Remark 3.7 (ii).

Remark 3.11. It is often asked whether for a given $w \in \partial I(S)$ one can find a Cahn-Hoffman vector field, i.e. a vector field $\xi$ on $S$ such that $\xi \in \partial \gamma(\mathbf{n}(P))$ for a.e. $P \in S$ and $\operatorname{div}_{S} \xi=w$. For a fully faceted surface we define $\xi$ by (3.6) on one $\gamma$-facet $F$ of $S$. Subsequently, for each $\gamma$-facet we repeat this procedure to get the Cahn-Hoffman vector field. Of course such a choice is not unique. It is possible to construct the Cahn-Hoffman vector fields even if there is a $\gamma$-directionally linear surface $L$ (satisfying assumptions of Lemma 3.8.). Here a difficulty arises when $L$ 
belongs to two different $\mathcal{O}$ 's partly because $\xi$ defined by (3.6) may have jump on $L$. Fortunately for the direction of $\nu, \partial \gamma(\mathbf{n}) \cdot \nu$ is a singleton so that $\xi \cdot \nu$ is continuous.

Remark 3.12. It is desirable to $\operatorname{define} \operatorname{div}_{S} \xi$ when $\xi \in \partial \gamma(\mathbf{n})$ is not necessarily Lipschitz continuous. According to geometric measure theory [35] the mean curvature $-\operatorname{div}_{S} \mathbf{n}$ is defined for any rectifiable set $S$, so in particular for a Lipschitz surface. But this is not enough to define $\operatorname{div}_{S} \xi$ in general (see Remark 3.7).

Remark 3.13. It is known that there is a 'stationary' crystalline surface such that its corners do not satisfy the compatibility condition " $\bigcap_{\mathbf{n}} \partial \gamma(\mathbf{n}) \neq \emptyset "$ ", where $\mathbf{n}$ is a normal of facets meeting at the corners; see Cahn and Taylor [10]. In this case, there are no continuous Cahn-Hoffman vector fields.

Remark 3.14. (Generalization) Our argument applies to more general energy of the form

$$
I(S)+\int_{D} g d x
$$

with constant $g$, where $D$ is a bounded set surrounded by $S$. It is not enough to replace the integrand in the definition $\varphi_{i}$ by $W_{i}(\nabla u)+g u$. If $g$ depends on the space variable, our argument does not apply directly since $\varphi_{i}$ may not be convex in $u$. We do not pursue this problem in this paper.

\section{Normal velocity and evolution equations}

We now consider a one parameter family $\left\{S_{t}\right\}_{t \in J}$ of an oriented Lipschitz (embedded) surfaces in $\mathbf{R}^{3}$, where $J$ is an interval. We say that $\left\{S_{t}\right\}_{t \in J}$ is $\mathcal{A}$-covered if there is an admissible coordinate system $\mathcal{A}=\left\{\mathcal{O}_{i}\right\}_{i=1}^{m}$ of $S_{t}$ for all $t \in J$ with $\left\{R_{i}\right\}_{i=1}^{m}$ independent of $S_{t}$. For an $\mathcal{A}$-covered $\left\{S_{t}\right\}_{t \in J}, R_{i}^{-1}\left(S_{t} \cap \mathcal{O}_{i}\right)$ is represented as the graph

$$
x_{3}=f_{i}\left(t, x_{1}, x_{2}\right),\left(x_{1}, x_{2}\right) \in \Omega_{i}
$$

of a function $f_{i}$ which is Lipschitz in the space variables. We say that $V^{t_{0}} \in L^{2}\left(S_{t_{0}}\right)$ is the normal velocity (field) of $S_{t}$ as $t=t_{0}$ if for each $i=1, \ldots, m$ the formula

$$
\sqrt{1+\left|\nabla f_{i}\left(t, x^{\prime}\right)\right|^{2}} V^{t_{0}}(P)=\partial_{t}^{+} f_{i}\left(t_{0}, x^{\prime}\right), x^{\prime}=\pi R_{i}^{-1} P
$$

holds for $\mathcal{H}^{2}$-almost every $P \in S_{t_{0}} \cap \mathcal{O}_{i}$ and the right derivative $\partial_{t}^{*} f_{i}\left(t_{0}, \cdot\right)$ exists as an element of $L^{2}\left(\Omega_{i}\right)$. This is a natural definition since the normal velocity of 
the graph $x_{3}=f_{i}\left(t, x^{\prime}\right)$ equals $\partial_{t} f_{i} /\left(1+\left|\nabla f_{i}\right|^{2}\right)^{1 / 2}$ if $f_{i}$ is sufficiently smooth. The normal velocity is unique if it exists. We need some regularity to define solutions of

$$
\beta(\mathbf{n}) V^{t} \in-\partial I\left(S_{t}\right) \text { on } S_{i}
$$

where $\beta$ is a positive continuous function on $S^{2}$, the unit sphere in $\mathbf{R}^{3}$.

Definition 4.1. For an open interval $J$ let $\left\{S_{t}\right\}_{t \in J}$ be $\mathcal{A}$-covered. Assume that the normal velocity $V$ exists for $t \in J$ as an element of $L^{2}\left(S_{t}\right)$. Assume that $f_{i}(t+s, \cdot) \rightarrow f_{i}(t, \cdot), \beta V^{t+s}\left(R_{i} \pi^{-1} x^{\prime}\right) \rightarrow \beta V^{t}\left(R_{i} \pi^{-1} x^{\prime}\right)$ in $L^{2}\left(\Omega_{i}\right)$ as $s \downarrow 0$ for $t \in J$. Here we set $\pi^{-1} x^{\prime}=R_{i}^{-1} P$, where $P \in S_{t} \cap \mathcal{O}_{i}$. Then we say that $\left\{S_{t}\right\}_{t \in J}$ is a $\varepsilon / t$ regular evolution.

Definition 4.2. Let $\left\{S_{t}\right\}_{t \in J}$ be a regular evolution on an open interval $J$. We say that $\left\{S_{t}\right\}$ is a strong solution of (4.1) if

$$
\beta(\mathbf{n}) V^{t} \in-\partial I\left(S_{t}\right) \text { for all } t \in J
$$

as an element of $L^{2}\left(S_{t}\right)$.

It appears that the velocity may not be determined. However, similarly to usual subdifferential equation $\partial u / \partial t \in-\partial \varphi(u)$ we have a characterization of the velocity.

Theorem 4.3. Assume that $\left\{S_{t}\right\}_{t \in J}$ is a strong solution of (4.1). Then $\beta(\mathbf{n}) V^{t_{0}}=$ $-\partial^{0} I\left(S_{t_{0}}\right)$ for each $t_{0} \in J$.

Proof. By Theorem $3.2 w_{1}=w_{2}$ holds outside $\gamma$-facets and $\gamma$-directionally linear surfaces of $S_{t_{0}}$ for $w_{1}, w_{2} \in \partial I\left(S_{t_{0}}\right)$. Thus it suffices to prove that

$$
\int_{\Sigma_{t_{0}}}\left|\beta(\mathbf{n}) V^{t_{0}}\right|^{2} d \mathcal{H}^{2} \leq \int_{\Sigma_{t_{0}}}|w|^{2} d \mathcal{H}^{2} \quad \text { for all } w \in-\partial I\left(S_{t_{0}}\right)
$$

where $\Sigma_{t_{0}}$ denotes the union of all $\gamma$-facets and $\gamma$-directionally linear surfaces of $S_{t_{0}}$.

Let $F$ be a facet of $S_{t_{0}}$ contained in $\mathcal{O}_{i}$. Later we shall suppress the index $i$ of $f_{i}$. We modify $f\left(t_{0}\right)=f\left(t_{0}, \cdot\right)$ outside $F$ so that $f\left(t_{0}+s, x^{\prime}\right)=\tilde{f} s\left(t_{0}, x^{\prime}\right)$ for every small $s>0$ and for a.e. $x^{\prime} \in \Omega_{i} \backslash \pi F_{\delta}$ and $\tilde{f}^{s}\left(t_{0}, x^{\prime}\right)=f\left(t_{0}\right)$ for $x^{\prime} \in \pi F$. Here $F_{\delta}$ is a $\delta$-neighborhood of $F$ in $S$. We may assume that $L^{2}\left(\Omega_{i}\right)$-limit of $\left(f\left(t_{0}+s\right)-\tilde{f}^{s}\left(t_{0}\right)\right) / s$ as $s \downarrow 0$ exists and it agrees with $f_{t}^{+}\left(t_{0}\right)$ on $\pi F$. We may also assume that $\tilde{f}^{s}$ belongs to the domain of $\varphi_{i}$ with the boundary condition $u=f\left(t_{0}+s\right)$. By the definition of geometric subdifferential of $I$ at $S_{t_{0}+s}$ the equation (4.1) yields

$$
\frac{1}{s}\left(\varphi_{i}\left(\tilde{f} s\left(t_{0}\right)\right)-\varphi_{i}\left(f\left(t_{0}+s\right)\right)\right) \geq \int_{\pi F_{\zeta}} \frac{\tilde{f} s\left(t_{0}\right)-f\left(t_{0}+s\right)}{s}\left(-\beta V^{t_{0}+s}\right) d x .
$$


Since $-w \in \partial I\left(S_{t_{0}}\right)$,

$$
\frac{1}{s}\left(\varphi_{i}\left(\tilde{f}\left(t_{0}+s\right)\right)-\varphi_{i}\left(f\left(t_{0}\right)\right)\right) \geq \int_{\pi F_{\delta}} \frac{\tilde{f}\left(t_{0}+s\right)-f\left(t_{0}\right)}{s}(-w) d x
$$

Here $\tilde{f}\left(t_{0}+s\right)$ is a modification of $f\left(t_{0}+s\right)$ such that $\tilde{f}\left(t_{0}+s\right)=f\left(t_{0}\right)$ outside $F_{\delta}$ and that $\tilde{f}\left(t_{0}+s\right)=f\left(t_{0}+s\right)$ on $F ; \tilde{f}\left(t_{0}+s\right)$ is chosen so that it belongs to the domain of $\varphi_{i}$ with boundary condition $u=f\left(t_{0}\right)$.

We may take $\tilde{f}^{s}, \tilde{f}$ defined in a suitable way so that

$$
\begin{aligned}
& \varphi_{i}\left(f\left(t_{0}\right)\right)-\varphi_{i}\left(\tilde{f}^{s}\left(t_{0}\right)\right)=o(s) \\
& \varphi_{i}\left(\tilde{f}\left(t_{0}+s\right)\right)-\varphi_{i}\left(f\left(t_{0}+s\right)\right)=o(s)
\end{aligned}
$$

provided that $\delta=\delta(s)$ is taken so that the area of $F_{\delta} \backslash F$ is $o(s)$. We can add up (4.3) and (4.4) and subsequently pass to the limit $s \rightarrow 0^{+}$. We come to

$$
\begin{aligned}
0 & \geq \int_{\pi F}\left(f_{t}^{+}\left(t_{0}\right) \beta V^{t_{0}}-f_{t}^{+}\left(t_{0}\right) w\right) d x \\
& =\int_{\pi F^{\prime}}\left\{\beta\left(V^{t_{0}}\right)^{2} \sqrt{1+|\nabla f|^{2}}-V^{t_{0}} \sqrt{1+|\nabla f|^{2}} w\right\} d x \\
& =\int_{F}\left\{\beta\left(V^{t_{0}}\right)^{2}-V^{t_{0}} w\right\} d \mathcal{H}^{2} .
\end{aligned}
$$

Hence,

$$
\int_{F} \beta\left(V^{t_{0}}\right)^{2} d \mathcal{H}^{2} \leq \int_{F} V^{t_{0}} w d \mathcal{H}^{2}
$$

Finally, with the help of the Schwarz inequality we can see that

$$
\int_{F}\left|\beta V^{t_{0}}\right|^{2} d \mathcal{H}^{2} \leq \int_{F}|w|^{2} d \mathcal{H}^{2}
$$

We argue in a similar way for directionally linear surfaces and obtain (4.2).

\section{Canonical restriction}

Since the velocity of solution of (4.1) is characterized by the canonical restriction of $\partial I(S)$ (Theorem 4.3), it is important to calculate $\partial^{0} I(S)$. Let $F$ be a $\gamma$-facet with Lipschitz boundary in a plane $H$ whose orientation (normal) is denoted by $\mathbf{n}_{F}$. Let $\nu_{F}$ be the outer normal of $\partial F$ as a set in $H$. We assume that $S$ is convex near $F$ in the direction of $\mathbf{n}_{F}$ so that $S$ is situated in one side of $H$ near $F$. In this situation by Lemma 3.3 and Theorems 3.2 and 3.6 it is easy to see that $w=\partial^{0} I(S)$ is a minimizer of the variational problem

$$
\|f\|_{L^{2}(F)}^{2}=\int_{F}|f|^{2} d \mathcal{H}^{2}
$$


among all $f \in K$, where

$$
\begin{aligned}
& K=\left\{f \in L^{2}(F) ; f=\operatorname{div}_{F} \xi \text { for some } \xi \in L^{\infty}\left(F ; \mathbf{R}^{3}\right)\right. \\
& \text { satisfying } \xi(x) \in \partial \gamma\left(\mathbf{n}_{F}\right) \text { for a.e. } x \in F \\
& \text { and } \left.\xi(x) \cdot \nu_{F}(x)=\sigma_{F}(x) \text { on } \partial F\right\} .
\end{aligned}
$$

Here $\sigma_{F}(x)=\sup \left(\partial \gamma\left(\mathbf{n}_{F}\right) \cdot \nu_{F}(x)\right)$. It is a kind of support function of the set $\partial \gamma\left(\mathbf{n}_{F}\right)$. Since $K$ is closed and convex in $L^{2}(F)$, there is a unique minimizer of (5.1) on $K$. The normal trace $\xi \cdot \nu$ is well-defined as an element of $H^{-1 / 2}(\partial F)$ since $\xi$ and $\operatorname{div}_{F}$ $\xi \in L^{2}(F)([41],[13])$.

It would be interesting to study the features of the minimizer. If $F=\partial \gamma\left(\mathbf{n}_{F}\right)$, then it is easy to see that $\xi=$ identity yields a minimizer and $\operatorname{div}_{F} \xi=2$. However, contrary to the case $n=2$ discussed in [12] it turns out that the minimizer might be nonconstant even if Frank $\gamma$ is polyhedron as proved by [43], [6]. On a directionally linear surface one can prove that the minimizer is a constant on a.e. line segment forming $L$ but we do not touch this problem here. We give here a criterion for constancy of the minimizer when $\partial \gamma\left(\mathbf{n}_{F}\right)$ is a unit disk due to [8], where a more general situation is studied.

Lemma 5.1([8]). Assume that $\partial \gamma\left(\mathbf{n}_{F}\right)$ is a unit disk. If

$$
\mathcal{H}^{1}(\partial A) / \mathcal{H}^{2}(A) \geq \mathcal{H}^{1}(\partial F) / \mathcal{H}^{2}(F)
$$

for all $A \subset F$, then the minimizer $f$ of (5.1) in $K$ is constant on $F$.

Corollary 5.2([8]). Condition (5.2) for all $A \subset F$ is equivalent to

$$
\max _{x \in \partial F} \kappa(x) \leq \mathcal{H}^{1}(\partial F) / \mathcal{H}^{2}(F)
$$

provided that $F$ is convex. Here $\kappa$ denotes the inward curvature of $\partial F$.

Since $\mathcal{H}^{1}(F) / \mathcal{H}^{2}(F)=2$ if $F$ is the unit disk, then condition (5.3) is fulfilled if $F$ is a small perturbation of a disk. For example, an ellipse with small eccentricity.

By rotation of coordinates we may assume that $\mathbf{n}_{F}=(0,0,1)$ so that $\partial \gamma\left(\mathbf{n}_{F}\right)=$ $W \times\{c\}$ with some $c \in \mathbf{R}$. Then the problem (5.2) is equivalent to minimizing

$$
\int_{F}|f|^{2} d x
$$

among all $f \in K^{\prime}$ with

$$
\begin{aligned}
& K^{\prime}=\left\{f \in L^{2}(F) ; \operatorname{div} \eta=f \text { for some } \eta \in L^{2}\left(F ; \mathbf{R}^{2}\right)\right. \\
& \text { satisfying } \eta(x) \in W \text { for a.e. } x \in F \\
& \text { and } \left.\eta(x) \cdot \nu_{F}(x)=\sup \left(W \cdot \nu_{F}\right)\right\} .
\end{aligned}
$$


If $\partial \gamma\left(\mathbf{n}_{F}\right)$ is a unit disk, we may assume that $W$ is a unit disk centered at zero in $\mathbf{R}^{2}$.

Lemma 5.3. Assume that $W$ is a closed unit disk in $\mathbf{R}^{2}$ centered at $0 \in \mathbf{R}^{2}$. If $f$ in $K^{\prime}$ is a constant on $F$, then there is a vector field $\eta$ on $F$ such that

a) $|\eta(x)| \leq 1$ for a.e. $x \in F$

b) $\eta \cdot \nu_{F}=1$ on $\partial F$

c) $f=\operatorname{div} \eta=\mathcal{H}^{1}(\partial F) / \mathcal{H}^{2}(F)$ (= constant denoted by $\left.v_{0}\right)$

Proof. Condition (a) is equivalent to saying that $\eta(x) \in W$ for a.e. $x \in F$; (b) follows from the boundary condition $\eta \cdot \nu_{F}=\sup \left(W \cdot \nu_{F}\right)$. If $\operatorname{div} \eta$ is a constant $v_{0}$, then integrating by parts yields

$$
v_{0} \int_{F} d x=\int_{\partial F} \nu_{F} \cdot \eta d \mathcal{H}^{1}=\int_{\partial F} 1 d \mathcal{H}^{1} .
$$

Thus $v_{0} \mathcal{H}^{2}(F)=\mathcal{H}^{1}(\partial F)$.

In [9] Cahn-Hoffman conjectured that if $f_{0}$ minimizes (5.4) in $K^{\prime}$, there is a gradient vector field $\nabla u$ such that $\eta=\nabla u$ and $\operatorname{div} \eta \in K^{\prime}$. Unfortunately, this conjecture is not true and we shall give a counterexample.

Proposition 5.4. Assume that $\eta \in L^{2}(F)$ satisfies a), b), c). Assume that $\partial F$ is smooth. Then there is no function $u$ that satisfies $\nabla u=\eta$ in $F$ unless $F$ is a disk.

Proof. If there were $u$ that satisfied $\nabla u=\eta$, then by b) and c) we would have

$$
\begin{aligned}
& \Delta u=v_{0}=\mathcal{H}^{1}(\partial F) / \mathcal{H}^{2}(F) \text { in } F \\
& \frac{\partial u}{\partial \nu_{F}}=1 \quad \text { on } \partial F
\end{aligned}
$$

Any solution $u$ to this equation is smooth in $F$ by elliptic regularity theory (e.g. [25]). It is also unique up to an additive constant. By a) and b) the value of $\eta$ on $\partial \Omega$ equals $\nu_{F}$ so that $\left.u\right|_{\partial F}$ is a constant. However, this is overdetermined and the only possibility is that $F$ is a disk according to a result of Serrin [34].

Theorem 5.5. Assume that $F$ is a convex $C^{2}$ set satisfying (5.3), e.g. an ellipse with small eccentricity, and assume that $F$ is not a disk. Let $f_{0}$ be the minimizer of (5.4) in $K^{\prime}$. Then there is no $u: \Omega \rightarrow \mathbf{R}$ that satisfies $\nabla u(x) \in W, \partial u / \partial \nu=1$ and $\xi=\nabla u$ and $f_{0}=\operatorname{div} \xi$.

This follows from Proposition 5.4 and Corollary 5.2. 
Acknowledgment. Much of this research was done during the visits of P. R. and M. P. to Hokkaido University. Its hospitality is gratefully acknowledged. The work of the first author was partly supported by a Grant-in-Aid for Scientific Research, No. 10304010, 12874024 the Japan Society of the Promotion of Science. The third author was in part supported by KBN grant 2 P03A 03517 and both of them enjoyed some support which was a result of Polish-Japanese Intergovernmental Agreement on Cooperation in the Field of Science and Technology. The second author was partly supported by the TMR (Training and Mobility of Researchers) program "Viscosity Solutions and their Applications".

\section{References}

[1] H. Attouch and A. Damlamian, Application des methods de convexité et monotonie a l'etude de certaines equations quasi lineares, Proc. Roy. Soc. Edinburgh 79A (1977), 107-129.

[2] F. Almgren and J. Taylor, Flat flow is motion by crystalline curvature for curves with crystalline energies, J. Differential Geometry 42 (1995), 1-22.

[3] S. B. Angenent and M. E. Gurtin, Multiphase thermomechanics with interfacial structure 2. Evolution of an isothermal interface, Arch. Rational Mech. Anal. 108 (1989), 323-391.

[4] G. Bellettini, R. Goglione and M. Novaga, Approximation to driven motion by crystalline curvature in two dimensions, preprint (1998).

[5] G. Bellettini and M. Novaga, Approximation and comparison for non-smooth anisotropic motion by mean curvature in $\mathbf{R}^{N}$, Math. Mod. Methods Appl. Sci., 10 (2000), 1-10.

[6] G. Bellettini, M. Novaga and M. Paolini, Facet-breaking for three-dimensional crystals evolving by mean curvature, Interfaces and Free boundaries, 1 (1999), 39-55.

[7] G. Bellettini, M. Novaga and M. Paolini, On a crystalline variational problem, preprint

[8] G. Bellettini, M. Novaga and M. Paolini, Characterization of facet-breaking for nonsmooth curvature flow in the convex case, preprint 
[9] J. W. Cahn and D. W. Hoffman, A vector thermodynamics for anisotropic surfaces -2. curved and faceted surfaces, Act. Metall. 22 (1974), 1205-1214.

[10] J. W. Cahn and J. Taylor, A contribution to the theory of surface energy minimizing shapes, Scripta Metall. 18 (1984), 1117-1120.

[11] C. M. Elliott, A. R. Gardiner and R. Schätzle Crystalline curvature flow of a graph in a variational setting, Adv. in Math. Sci. Appl. 8 (1998), 425-460.

[12] T. Fukui and Y. Giga, Motion of a graph by nonsmooth weighted curvature, In: World congress of nonlinear analysts '92 (ed. V. Lakshmikantham), Walter de Gruyter, Berlin I, (1996), 47-56.

[13] D. Fujiwara and H. Morimoto, An $L_{r}$-theorem of the Helmholtz decomposition of vector fields, J. Fac. Sci. Univ. Tokyo, Sect. 1A Math., 24 (1977), 685-700.

[14] M.-H. Giga and Y. Giga, Consistency in evolutions by crystalline curvature, In: Free Boundary Problems, Theory and Applications (eds. M. Niezgódka and P. Strzelecki), Proceedings of the Zakopane Congress '95, Pitman Research Notes in Mathematics Series 363(1996), pp.186-202, Longman.

[15] M.-H. Giga and Y. Giga, Evolving graphs by singular weighted curvature, Arch. Rational. Mech. Anal. 141 (1998), 117-198.

[16] M.-H. Giga and Y. Giga, A subdifferential interpretation of crystalline motion under nonuniform driving force, Proc. of the International Conference in Dynamical Systems and Differential Equations, Springfield Missouri, (1996), "Dynamical Systems and Differential Equations", (eds. W.-X. Chen and S.-C. $\mathrm{Hu})$, Southwest Missouri Univ. vol.1(1998), pp.276-287

[17] M.-H. Giga and Y. Giga, Stability for evolving graphs by nonlocal weighted curvature, Commun. in Partial Differential Equations 24 (1999), 109-184.

[18] M.-H. Giga and Y. Giga, Remarks on convergence of evolving graphs by nonlocal curvature, In: Progress in Partial Differential Equations vol.1 (eds. H. Amann, C. Bandle, M. Chipot, F. Conrad and L. Shafrir) Pitman Research Notes in Mathematics Series, 383 (1998), pp.99-116, Longman, Essex, England

[19] M.-H. Giga and Y. Giga, Crystalline and level set flow - Convergence of a crystalline algorithm for a general anisotropic curvature flow in the plane, In: Free Boundary Problems, Theory and Applications (ed. N. Kenmochi), GAKUTO 
International Series, Mathematical Sciences and Applications 13 (2000), pp. 64-79, Gakuto.

[20] M.-H. Giga and Y. Giga, Generalized motion by nonlocal curvature in the plane, preprint.

[21] M.-H. Giga, Y. Giga and R. Kobayashi, Very singular diffusion equations, Proc. of Taniguchi Conf. on Mathematics, Nara 98 (ed. T. Sunada), Advances Studies in Pure Mathematics 26 (2000), to appear.

[22] Y. Giga and M. Gurtin, A comparison theorem for crystalline evolution in the plane, Quart. of Appl. Math. 54 (1996), 727-737.

[23] Y. Giga, M. E. Gurtin and J. Matias, On the dynamics of crystalline motions, Japan J. Indust. Appl. Math. 15 (1998), 7-50.

[24] Y. Giga and R. Schätzle, Uniform approximation for anisotropic curvature flow by the Allen-Cahn equations, in preparation

[25] D. Gilbarg and N. Trudinger, Elliptic Partial Differential Equations of Second Order, 2nd edition, Springer (1983).

[26] P. M. Girão, Convergence of a crystalline algorithm for the motion of a simple closed convex curve by weighted curvature, SIAM J. Numer. Anal. 32 (1995), 886-899.

[27] P. M. Girão and R. V. Kohn, Convergence of a crystalline algorithm for the heat equation in one dimension and for the motion of a graph by weighted curvature, Numer. Math. 67 (1994), 41-70.

[28] D. W. Hoffman and J. W. Cahn, A vector thermodynamics for anisotropic surfaces -1. Fundamentals and applications to plane surface junctions, Surface Sci. 31 (1972), 368-388.

[29] K. Ishii and H.-M. Soner, Regularity and convergence of crystalline motion, SIAM J. Math. Anal. 30 (1999), 19-37.

[30] R. Kobayashi and Y. Giga, Equations with singular diffusivity, J. Stat. Phys. 95 (1999), 1187-1220.

[31] P. Rybka, A crystalline motion : uniqueness and geometric properties, SIAM J. Appl. Math. 57 (1997), 53-72. 
[32] P. Rybka, Crystalline version of the Stefan problem with Gibbs- Thompson law and kinetic undercooling, Adv. Differential Equations, 3 (1998), 687-713.

[33] P. Rybka, The crystalline version of the modified Stefan problem in the plane and its properties, SIAM J. Math. Anal. 30 (1999), 756-786.

[34] J. Serrin, A symmetry problem in potential theory, Arch. Rational Mech. Anal. 43 (1971), 304-318.

[35] L. Simon, Lectures on Geometric Measure Theory, Proc. Centre for Math. Anal., Australian Nat. Univ. 3 (1983).

[36] A. Stancu, Uniqueness of self-similar solutions for a crystalline flow, Indiana Univ. Math. J. 45 (1996), 1157-1174.

[37] A. Stancu, Asymptotic behavior of solutions to a crystalline flow, Hokkaido Math. J. 27 (1998), 303-320.

[38] J. Taylor, Constructions and conjectures in crystalline nondifferential geometry, In: Differential Geometry (eds. B. Lawson and K. Tanenblat), Proceedings of the Conference on Differential Geometry, Rio de Janeiro, Pitman Monographs Surveys in Pure and Applied Math. 52 (1991) pp.321-336, Pitman, London

[39] J. Taylor, Mean curvature and weighted mean curvature, Acta Metall. 40 (1992), 1475-1485.

[40] J. Taylor, Motion of curves by crystalline curvature, including triple junctions and boundary points, In: Differential Geometry: Partial Differential Equations on Manifolds (eds. R. Greene and S. T. Yau), Proc. Symp. Pure Math., 54(1993) Part I, pp.417-438, Amer. Math. Society Providence, RI

[41] R. Temam, Navier-Stokes Equations, North Holland, Amsterdam, 1977

[42] J. Taylor and J. W. Cahn, Diffuse interfaces with sharp corners and facets : Phase field models with strongly anisotropic surfaces, Physica D 112 (1998), 381-411.

[43] J. Yunger, Facet stepping and motion by crystalline curvature, PhD Thesis, Rutgers University (1998). 